\title{
Necesidades educativas especiales en niños y adolescentes con diagnóstico de diabetes
}

\author{
Special educational needs of children and adolescents with diabetes diagnosis
}

\author{
Ismael García Cedillo \\ Karla Lozano Cabral
}

\begin{abstract}
RESUMEN
La diabetes pediátrica puede tener serias consecuencias físicas y, con ello, comprometer la trayectoria escolar de los estudiantes. El propósito del presente estudio fue determinar si los alumnos con diagnóstico de diabetes mellitus presentan necesidades educativas especiales y si estas están siendo atendidas por sus maestros. Participaron nueve niños y adolescentes con diabetes mellitus tipo 1 y 2 , entre los 10 y 17 años, quienes recibían atención médica en un hospital público de la ciudad de San Luis Potosí, México; también se trabajó con sus padres, docentes y un compañero de cada alumno. Se hicieron breves entrevistas estructuradas a los padres, a los docentes y a los compañeros de los participantes; se aplicó la Escala Wechsler de Inteligencia para Niños a los alumnos, el cuestionario Estrategias para Fortalecer el Aprendizaje de Mitchell y la Guía de Prácticas Inclusivas en el Aula, versión de observación a sus docentes, además se revisaron sus boletas de calificaciones. Se encontró que solamente dos alumnos califican para considerar que presentan necesidades educativas especiales, sin estar asociadas a la diabetes mellitus, sino a sus condiciones personales y a la situación socioconómica de la familia; las escuelas no parecen atender apropiadamente dichas necesidades.
\end{abstract}

Palabras claves: enfermedades crónicas, integración educativa, necesidades de apoyo.

\begin{abstract}
Diabetes in pediatric ages can have serious physical consequences and, consequently, compromise the students' academic trajectory. The purpose of the study was to identify if students with diabetes mellitus diagnosis present special educational needs. Nine children and adolescents with type 1 and 2 diabetes mellitus, between the ages of 10 and 17, who were receiving medical attention in a public hospital in the city of San Luis Potosí (Mexico) participated; alongside their parents, teachers, and a classmate of each student. Brief interviews to parents, teachers and schoolmates were done; the Wechsler Intelligence Scale for Children (WISC-IV) were applied to participants. Also, the Survey on Strategies for Strengthening Learning and the Guide for the Evaluation of Inclusive Practices in the Classroom were applied to teachers. The students' grades were registered. It was found that only two students qualify to be considered to have special educational needs; their needs were not attended by their teachers.
\end{abstract}

Keywords: chronic diseases, educational integration, support needs. 


\section{INTRODUCCIÓN}

Las enfermedades crónicas han aumentado en la población mundial, lo cual incluye a los niños y adolescentes ( $\mathrm{NyA}$ ); la diabetes mellitus (DM) es una de ellas (OMS, 2016). La DM puede afectar en algunos casos el desarrollo del NyA en áreas tanto académicas como emocionales (Hawrylak y Grau, 2014). Por lo anterior, es necesario conocer las consecuencias de la enfermedad en la trayectoria escolar de NyA con diabetes para determinar qué medidas pueden implementarse para atender las posibles necesidades educativas especiales (NEE) asociadas a este padecimiento.

Según la Federación Internacional de Diabetes (FID, 2018), a nivel mundial hay 425 millones de personas con DM, lo cual representa el 8.8\% de la población entre 20 y 79 años. Los países con mayor número de personas con DM son: China, India, EUA, Brasil, México e Indonesia. Se proyecta que para el año 2045 México se posicione en cuarto lugar mundial. En América se calcula que hay 72 millones de personas y en México 12 millones de personas de 20 a 79 años que presentan DM. Preocupa que poco más de 212 millones de personas desconozcan que tienen DM, ya que al ignorar su situación no previenen el desarrollo progresivo de las complicaciones asociadas a este padecimiento.

En relación con los NyA, la FID (2015) calcula que, a nivel mundial, hay una prevalencia de 542 mil niños entre los 0 y 14 años con DM tipo 1. En México, la Encuesta Nacional de Salud y Nutrición Ensanut (Instituto Nacional de Salud Pública, 2012) señala que el $0.68 \%$ de NyA entre 10 y 19 años padece esta enfermedad, lo cual involucra a 155 mil mexicanos. De acuerdo con el Sistema Único de Información para la Vigilancia Epidemiológica (Secretaría de Salud, 2019), hay 10,414 casos de diabetes mellitus en San Luis Potosí, México, estado donde se hizo la presente investigación.

En la DM, la producción de insulina por parte del páncreas es insuficiente o el organismo es incapaz de metabolizarla adecuadamente (FID, 2015). La OMS (2016) clasifica a la DM de tres maneras: tipo 1, tipo 2 y gestacional; su tratamiento consiste en la inyección de insulina o ingesta de fármacos, una alimentación sana y baja en

Ismael García Cedillo. Profesor-Investigador de Tiempo Completo en la Facultad de Psicología de la Universidad Autónoma de San Luis Potosí, México. Es doctor en Psicología Clínica por la Facultad de Psicología de la UNAM. Fue director del Proyecto Nacional de Integración Educativa (1998-2001). Entre sus últimas publicaciones se encuentra el artículo "Estrategias educativas inclusivas y su relación con la autoeficacia de docentes en formación” (2019). Es miembro del Sistema Nacional de Investigadores, Nivel 2, Perfil Prodep e integrante del Cuerpo Académico Consolidado Evaluación e Intervención en Psicología y Educación. Correo electrónico: garcia.ismael52@gmail.com. ID: https://orcid.org/0000-0002-4820-5633.

Karla Lozano Cabral. Universidad Autónoma de San Luis Potosí, México. Es maestra en Psicología por la Facultad de Psicología de la UASLP. Fue profesora y asesora en la Universidad Tec Milenio, del Centro de Recursos e Información para la Integración Educativa (CRIE), maestra del Centro de Atención Múltiple (CAM) del Sistema Educativo Estatal Regular (SEEP) y maestra del idioma inglés dentro del Programa Nacional de Inglés en Educación Básica. Correo electrónico: karla.regis@hotmail.com. ID: https:// orcid.org/0000-0003-1301-1545. 
carbohidratos simples y ejercicio diario. Debido a que el tratamiento implica la modificación de hábitos relacionados con el estilo de vida, la adherencia al tratamiento puede representar un desafío para el NyA y su grupo familiar.

Grau (2003) menciona que cuando a la familia se le informa que el NyA presenta una enfermedad crónica, como la DM (o cualquier otra), sus integrantes habitualmente atraviesan una crisis: los padres pueden negar la enfermedad, sentirse culpables o presentar ansiedad por el futuro de sus hijos; los hermanos pueden sentir preocupación o molestia, y los más pequeños pueden presentar conductas regresivas y buscar la atención desplazada hacia el NyA con diabetes. Finalmente, el NyA enfermo tendrá que afrontar las hospitalizaciones y las secuelas de la enfermedad.

Debido a la cronicidad del padecimiento, un NyA con DM debe aprender a vivir con ella y realizar adaptaciones a nivel individual, familiar y escolar (FID, 2015). La postura que tomen los padres y los docentes influye en el impacto del diagnóstico de la enfermedad y sus secuelas en la vida del NyA. Es necesario, por tanto, que exista una comunicación fluida y constante entre estos tres actores, con el fin de proporcionar los apoyos que requiere el NyA. Bazán (2012) considera necesario ofrecer información sobre las características de la enfermedad, las secuelas, los cuidados necesarios y el tipo de tratamiento que se prescribió, tanto al niño como a su entorno familiar y escolar.

\section{NeCEsidades EDUCATIVAS ESPECIALES}

En el presente estudio se abordó la problemática de los NyA con DM desde el punto de vista escolar, esto es, se buscó identificar si presentan necesidades educativas especiales (NEE).

El concepto de NEE apareció por primera vez en 1978 en el Informe Warnock (Montero, 1991) y se relaciona con el proceso de integración educativa, esto es, con la atención educativa de los niños con NEE en la escuela regular y no en escuelas especiales. En el Marco de Acción de la Declaración de Salamanca (UNESCO, 1994) se mencionó que "el término NEE se refiere a las necesidades de NyA que se derivan de su capacidad o sus dificultades de aprendizaje. Muchos niños experimentan dificultades de aprendizaje y tienen, por lo tanto, NEE en algún momento de su escolarización” (p. 6).

Una definición de las NEE menciona que constituyen los apoyos que deben recibir algunos NyA para optimizar sus aprendizajes (García, Escalante, Escandón, Fernández, Mustri y Puga, 2000). Las NEE pueden ser transitorias o permanentes. Entonces, un NyA con NEE es aquel que presenta alguna dificultad de aprendizaje a lo largo de su escolarización y, por lo tanto, requiere de atención y recursos educativos específicos, distintos de los que necesitan sus compañeros (García, Fernández y Medina, 2010). 
Las NEE pueden deberse a los siguientes factores: a) personales, como tener alguna discapacidad, enfermedad o por presentar capacidades y aptitudes sobresalientes; b) sociales, como vivir con una familia disfuncional, anómica o en la que se presentan abusos físicos o sexuales, y c) escolares, como asistir a una escuela con muy pocos recursos o por tener un maestro poco preparado (García et al., 2000).

En concordancia con lo anterior, pero en relación con las enfermedades crónicas, Grau (2003) refiere que son tres los tipos de NEE asociadas a estas: las derivadas del diagnóstico y tratamiento de la enfermedad; las derivadas de los trastornos emocionales y sociales, y las relacionadas con el currículo escolar.

Se debe tomar en cuenta que los sistemas educativos hoy en día tienen como reto trascender la integración educativa para implementar procesos de educación inclusiva, reto cuyas metas son muy ambiciosas (Acedo, 2008; Ainscow, 2001; López, 2012). Entre otras cosas, la educación inclusiva busca eliminar las barreras para el aprendizaje y la participación que enfrenta el alumnado, concepto que busca sustituir al de NEE. Sin embargo, diversos autores han señalado su utilidad y se han opuesto a su eliminación (García, 2015; Norwich, 2010).

No se puede pasar por alto que, en el país, se ha dado un fuerte debate entre quienes se manifiestan a favor de implementar la educación inclusiva de manera radical (lo que, como se mencionó, implicaría el abandono del concepto de NEE) y quienes plantean que se deben tomar en cuenta las necesidades muy específicas de algunos alumnos, pues su satisfacción es imprescindible para garantizar una trayectoria escolar exitosa (García, 2018). En México recientemente se presentó la Estrategia Nacional de Educación Inclusiva, a la cual se le considera como "uno de los elementos que materializa el Acuerdo Educativo Nacional que representa el nuevo artículo 3o. de la Constitución Política de los Estados Unidos Mexicanos" (SEP, 2020, p. 5). Mediante la educación inclusiva se busca, entre otros objetivos, la realización de ajustes razonables, esto es, las "modificaciones y adaptaciones necesarias y adecuadas que no impongan una carga desproporcionada o indebida, cuando se requieran en un caso particular, para garantizar a las personas el goce o ejercicio... de todos los derechos humanos..." (p. 10). En otras palabras, en la mencionada estrategia se destaca la necesidad de proporcionar apoyos específicos a algunos alumnos, con lo cual se ratifica la necesidad de utilizar el concepto de NEE.

Ahora bien, la educación inclusiva busca ofrecer una educación de calidad a todos los estudiantes, con énfasis en aquellos que pertenecen a grupos en condición de vulnerabilidad. Para garantizar la calidad de la educación, el profesorado debe estar preparado, lo que implica, entre otros aspectos, ser capaz de emplear metodologías de enseñanza diversificadas y de trabajar de manera colaborativa inter y multidisciplinariamente con diversos profesionales, para dar así respuesta a las NEE que pudieran presentar algunos de sus alumnos (García, 2015). En este sentido, es importante que el docente esté en constante actualización y que cuente con recursos 
y apoyos con el fin de atender la diversidad de alumnos (Adame, 2015). Asimismo, los docentes deben responder con una actitud positiva ante las dificultades que se les puedan presentar, lo que implica ver a los problemas de sus alumnos como un reto y no como un problema (Villa, 2011).

Por lo anterior, es muy importante que los padres de un NyA con DM tomen la iniciativa para informar a la escuela, al inicio del ciclo escolar o al momento de debut de la DM, sobre la condición de su hijo, con la finalidad de hacerla partícipe $y$, por consiguiente, lograr que el alumno alcance un mejor control de la enfermedad (Gómez-Rico, Pérez-Martin y Montoya-Castilla, 2015). De hecho, se puede ir más allá, pues se puede gestionar que algún integrante del equipo médico proporcione orientación a maestros, padres y a los mismos alumnos, sobre los cuidados que requiere el NyA con diagnóstico de diabetes.

Un docente bien informado puede desempeñar un papel importante en el proceso de comprensión y asimilación de la DM, además de que puede motivar al alumno para que participe en todas las actividades de la escuela (Bazán, 2012). Villa Martín (2011) considera imprescindible que el docente valore siempre los aspectos positivos de su alumno, que le fomente seguridad y procure la aceptación de sus compañeros. Además, cuando el docente tiene conocimientos sobre la enfermedad del alumno, puede planear y realizar ajustes en las actividades escolares.

\section{Antecedentes}

De acuerdo con Quintero y Melo (2012), el rendimiento escolar del alumno en la escuela depende de varios factores. En la escuela, influyen las metodologías de los docentes, su preparación profesional, las condiciones físicas del aula, la cantidad de materiales didácticos con que cuentan, el uso eficaz del tiempo, las características de sus compañeros, las dinámicas que se generan en el grupo, su círculo de amistades y el cambio de nivel educativo, entre otros. En la familia, influye el apoyo brindado a los estudios del hijo, los recursos económicos y el nivel de estudios alcanzado por los miembros de la familia. A nivel personal, pueden influir las características del niño, su motivación para el aprendizaje, sus posibles trastornos psicológicos, enfermedades y su nivel de desarrollo.

Al hablar sobre rendimiento escolar asociado a enfermedades crónicas, o específicamente con la DM, se encuentran dos posturas. Algunos autores refieren que las enfermedades crónicas traen consigo alteraciones cognitivas o, de manera más amplia, psicológicas (García, Hernández y Silva, 2019; Grau y González, 2001; Northam, Anderson, Jacobs, Hughes, Warne y Werther, 2001; Villa, 2011), mientras que otros descartan esta idea (Chamorro, Badajoz y Luzuriaga, 2002; Chamorro, Martínez y Tomás, 2002; Fernández, Pérez y Arias, 2010; McCarthy, Lindgren, Mengeling, Tsalikian y Engvall, 2002). 
Manchón, Carrasco, Fernández, Fernández y Frías (2009) refieren que la mayoría de los padres y docentes consideran que la integración y la aceptación escolar de los NyA con DM son buenas. La FID (2015) encontró que los padres consideran que la adaptación a la vida escolar es buena antes y después del diagnóstico, siendo el tutor, los docentes y los compañeros los principales apoyos de los NyA. También encontraron que los NyA con DM necesitan contar con docentes mejor informados y que es conveniente la presencia de un enfermero en el interior de la escuela.

En lo que concierne a las aportaciones que abordan el área psicológica, Bazán (2012) señala que la aparición de problemas psicológicos dependerá de los recursos del NyA y de la familia para hacer frente a la aparición de la DM. Bilbao-Cercós, Beniel-Navarro, Pérez-Marín, Montoya-Castilla, Alcón-Sáez y Prado-Gascó (2014) encontraron en su estudio que el 50\% de NyA entre ocho y 16 años con DM tipo 1 mostró tener un autoconcepto bajo y un 30\% un autoconcepto muy bajo. GómezRico, Pérez-Martin y Montoya-Castilla (2015) encontraron que las restricciones y exigencias del tratamiento inducen a la presencia de síntomas de depresión, ansiedad, distorsión en la autoimagen, baja autoestima y trastornos alimenticios; refieren además la necesidad de tomar acciones de autogestión, autoeficacia y competencia social para adaptarse y hacer frente a la enfermedad.

En resumen, la evidencia actual no es concluyente sobre la aparición de NEE asociadas al diagnóstico de DM en NyA y, por lo tanto, sobre la necesidad o no de implementar apoyos específicos para que los alumnos alcancen un buen desarrollo de su trayectoria escolar. En México no se encontraron estudios publicados en revistas de prestigio sobre este tema.

Por lo anterior, el objetivo general del presente estudio es determinar si la DM se asocia con la aparición de NEE en NyA y su posible atención en las escuelas. Los objetivos específicos son: a) determinar si el diagnóstico de DM se relaciona con la aparición de las NEE, y b) identificar si las escuelas atienden las NEE que probablemente presentan algunos NyA con DM.

\section{Método}

\section{Diseño}

Se trata de un estudio descriptivo, de un solo grupo (Hernández, Fernández y Baptista, 2010).

\section{Población}

La población estuvo formada por nueve NyA con DM tipos 1 y 2, entre los 5 y 17 años, que cursaban la educación básica o media superior, quienes se encontraban recibiendo atención médica en un hospital público de la ciudad de San Luis Potosí, México. 


\section{Criterios de inclusión}

Tener diagnóstico de DM tipo 1 o 2, estar escolarizado, asistir al servicio de pediatría del hospital, tener entre 5 y 17 años de edad y la aceptación de la participación voluntaria en el estudio por parte de los padres y del NyA.

\section{Muestra}

Se invitó a participar a todos los sujetos de la población con DM que asistía al hospital; la muestra quedó conformada por nueve NyA con DM tipos 1 y 2, con edades de 10 a 16 años, con firma de consentimiento informado por parte de sus padres y la aceptación de los mismos pacientes. Asimismo participaron nueve padres de familia, nueve docentes y nueve compañeros de grupo. En la tabla 1 se describen el sexo, edad y nivel educativo de los participantes, así como algunas características de su enfermedad. Se optó por presentar estos datos más adelante, para que se puedan asociar con mayor facilidad con los datos de las demás tablas.

\section{INSTRUMENTOS Y PROCEDIMIENTOS}

Se realizaron breves entrevistas a los padres, maestros y compañeros de los participantes para conocer algunos datos personales y algunas opiniones con respecto a los NyA.

Con el fin de identificar si los maestros de los participantes desarrollaban prácticas inclusivas en sus aulas y, con ello satisfacer las necesidades educativas especiales de algunos de sus estudiantes, se aplicó a los docentes la Guía de Evaluación de las Prácticas Inclusivas en el Aula, formato de observación (GEPIA-O) (García, Romero y Escalante, 2011; García, Romero, Escalante y Flores, 2018); este instrumento permite conocer en qué áreas se implementan prácticas inclusivas. La GEPIA-O está compuesta por ocho categorías y tiene un total de 45 reactivos en escala Likert, con valores del 1 al 4, con opciones de respuesta: totalmente falso, parcialmente falso, parcialmente cierto y totalmente cierto. Sus niveles de confiabilidad ( $\alpha=0.834$ ) y validez aparente y de contenido son apropiados para utilizar esta escala en poblaciones escolares mexicanas (García et al., 2018).

Se pidió a los docentes contestar el cuestionario Estrategias para Fortalecer el Aprendizaje (su nombre en inglés es What really works in special and inclusive education, de Mitchell [2014], el cual fue traducido por García, Romero y Fletcher [2015]), con el fin de indagar las prácticas que realizan los docentes para fortalecer los aprendizajes de sus alumnos. El cuestionario está compuesto por ocho dimensiones y tiene un total de 34 reactivos, los cuales se responden mediante una escala Likert, con valores del 1 al 4, con las siguientes opciones de respuesta: nunca, pocas veces, a menudo y muchas veces. En México, Ramos-Estrada, García-Cedillo, Sotelo-Castillo, LópezValenzuela y Murillo-Parra (2020) hicieron un estudio para investigar su confiabilidad ( $\alpha$ superior o igual a .709) y su validez factorial (mediante el análisis factorial explora- 
torio, que validó cinco de las subescalas), encontrando valores muy apropiados para ambas dimensiones.

A los NyA se les aplicó la WISC-IV, la cual fue estandarizada en México (SánchezEscobedo, 2006; Wechsler, Flanagan y Kaufman, 2007), con el objetivo de evaluar el nivel de inteligencia de NyA. La prueba está conformada por 15 subpruebas, de las cuales 10 son obligatorias y 5 optativas; los resultados de estas pruebas conforman cuatro índices: comprensión verbal (CV), razonamiento perceptual (RP), memoria de trabajo (MT) y velocidad de procesamiento (VP) que, en conjunto, indican el coeficiente intelectual total (CIT). Para el presente estudio se aplicaron 13 subpruebas para conocer el nivel de conocimientos y el rendimiento académico de los $\mathrm{NyA}$. Se evaluaron los resultados individuales de cada subprueba, de cada índice y el CIT.

Se solicitó a las escuelas las boletas de calificaciones pre y post diagnóstico de la enfermedad. El rendimiento académico habitualmente se mide mediante las calificaciones escolares (Quintero y Melo, 2012), ya que son un reflejo de los conocimientos aprendidos (Cascón, 2000). En el presente estudio se hizo una comparación entre las calificaciones previas al diagnóstico y las del más reciente ciclo escolar, capturadas de las boletas de calificaciones.

Además se solicitó al equipo médico los siguientes indicadores biológicos: el índice de masa corporal (IMC, los valores normales van de 18 a 25), para tener un indicador del nivel nutricional de los NyA, y los valores de la hemoglobina glucosilada (de acuerdo con Galván, ${ }^{1}$ los valores normales de este análisis para niños mayores a 12 años deben ser menores de 7\%; en niños menores deben ser menores de $7.5 \%$ [Galván, E., comunicación personal, 18 de noviembre del 2018], indicador muy importante del nivel de control de la enfermedad).

Tabla 1. Características de la muestra.

\begin{tabular}{ccccccccccc}
\hline & Sexo & Edad & $\begin{array}{c}\text { Tipo } \\
\text { de DM }\end{array}$ & $\begin{array}{c}\text { Tiempo con } \\
\text { DM (meses) }\end{array}$ & $\begin{array}{c}\text { Nivel } \\
\text { educativo }\end{array}$ & IMC & Clasificación & HbA1c & de HbA \\
\hline 1 & F & 14 & 2 & 4 & Secundaria & 28.8 & Obesidad & 6.90 & Normal \\
\hline 2 & F & 14 & 2 & 18 & Secundaria & 32 & Obesidad & 5.50 & Normal \\
\hline 3 & M & 12 & 1 & 4 & Secundaria & 32.1 & Obesidad & 10.4 & Alta \\
\hline 4 & F & 10 & 1 & 9 & Primaria & 16.8 & Normal & 7.70 & Normal \\
\hline 5 & F & 16 & 1 & 36 & Preparatoria & 23.7 & Normal & Un poco alta \\
\hline 6 & F & 14 & 1 & 6 & Secundaria & 17.7 & Normal & 8.00 & Un poco alta \\
\hline 7 & M & 14 & 1 & 14 & Secundaria & 27.7 & Sobrepeso & 7.40 & Normal \\
\hline 8 & F & 12 & 2 & 44 & Secundaria & 25.5 & Sobrepeso & 12.7 & Muy alta \\
\hline 9 & M & 15 & 2 & 8 & Preparatoria & 29.8 & Obesidad & 6.00 & Normal \\
\hline
\end{tabular}

Fuente: Elaboración propia.

1 Médico encargado del servicio de pediatría del hospital donde se realizó la investigación. 
En relación con la muestra, se podrá apreciar en la tabla 1 que, de los nueve NyA, cinco presentan DM tipo 1 y cuatro DM tipo 2; seis son mujeres y tres son hombres; uno asiste a primaria, seis a secundaria y dos están en preparatoria. El rango de edad de las personas participantes oscila entre los 10 y los 16 años, con una media de 13 años. En cuanto al tiempo con el diagnóstico, cinco de ellos tienen menos de un año, dos tienen entre uno y dos años, uno tiene tres años y uno más tiene tres años y medio. En relación con el IMC, dos NyA presentan sobrepeso, cuatro obesidad y tres tienen valores promedio. En cuanto a la hemoglobina glucosilada, cinco NyA tienen valores promedio, dos la tienen un poco alta y dos alta o muy alta.

\section{Procedimiento}

1. Se entregó el anteproyecto al hospital para su revisión por parte del Comité de Ética; una vez aprobado, se procedió a iniciar el estudio.

2. Se invitó a los padres de familia de los pacientes y a los $\mathrm{NyA}$ a participar en el proyecto. Se solicitó el consentimiento informado de los padres por escrito y de los alumnos de manera verbal.

3. Se solicitó los valores del IMC y de la hemoglobina glucosilada; también se realizaron las entrevistas al padre o tutor y se aplicó la WISC-IV a los NyA.

4. Se pidió la autorización por escrito a los padres y verbal a los $\mathrm{NyA}$ para ir a sus escuelas; una vez obtenida, se pidió la aprobación de la escuela para realizar el estudio. En las escuelas, solamente se informó al director del propósito de la investigación, pues era indispensable preservar la confidencialidad con respecto al diagnóstico de los alumnos; el director también autorizó la realización de las observaciones y entrevistas a maestros y alumnos. A los docentes se les comentó que se realizarían observaciones sobre sus prácticas. Se aplicó una breve entrevista a un compañero de grupo del $\mathrm{NyA}$, el cual fue seleccionado de la lista de asistencia, para igualarlo en edad y sexo con el alumno participante y a los docentes, a estos últimos también se les aplicó el cuestionario Estrategias para Fortalecer el Aprendizaje y la GEPIA-O.

5. Finalmente se gestionó en la dirección de las escuelas el acceso a las boletas de calificaciones previas y posteriores al diagnóstico de la DM2.

\section{Procesamiento de datos y análisis estadístico}

Para el cuestionario Estrategias para Fortalecer el Aprendizaje, la GEPIA y la WISC IV se utilizó estadística descriptiva. No se utilizó estadística inferencial por el número reducido de casos.

Las entrevistas se codificaron de acuerdo con las principales categorías que emergieron en las respuestas. 


\section{Consideraciones éticas}

De acuerdo con lo señalado en el artículo 17 del Reglamento de la Ley General de Salud en Materia de Investigación para la Salud (Presidencia de la República, 1986), el presente estudio fue una investigación sin riesgo para los participantes. Los procedimientos utilizados en la investigación se apegan a las normas éticas estipuladas en dicho documento y en los principios de la Declaración de Helsinki de la Asociación Médica Mundial (2013[1964]).

No se pueden generalizar los resultados del presente estudio porque la muestra fue muy reducida. Además, faltó mayor colaboración de una escuela para aplicar todos los instrumentos de evaluación. Por otro lado, aunque las calificaciones pueden ser un buen indicador del rendimiento académico de los alumnos, probablemente es insuficiente, tal vez se requieren evaluaciones adicionales.

\section{Resultados}

Con el fin de determinar la presencia de las NEE en los alumnos, se revisaron las calificaciones de las boletas escolares; esta información se trianguló con las puntuaciones del WISC-IV y con la opinión de los padres, docentes y compañeros.

En la tabla 2 se muestra la edad, las calificaciones anteriores y posteriores al inicio de la enfermedad y su comparación, el tiempo de meses con DM y el CIT de la WISC-IV.

Como se muestra en la tabla 2, después del diagnóstico de DM tres $\mathrm{NyA}$ (casos 3, 6 y 9) subieron ligeramente sus calificaciones, dos (casos 7 y 8) las mantuvieron, en otros dos (casos 1 y 5) bajaron ligeramente y, por último, dos de ellos (casos 2 y 4) bajaron en mayor medida. También se podrá apreciar en la tabla 2 que los meses cursados con la enfermedad no parecen asociarse a las calificaciones, pues el sujeto 8

Tabla 2. Comparaciones de calificaciones de los NyA, pre y post al inicio de la enfermedad.

\begin{tabular}{|c|c|c|c|c|c|c|}
\hline ID & Edad & $\begin{array}{l}\text { Tiempo (meses) } \\
\text { con DM }\end{array}$ & $\begin{array}{l}\text { Promedio de calificaciones } \\
\text { (previas al Dx) }\end{array}$ & $\begin{array}{c}\text { Calificaciones } \\
\text { actuales }\end{array}$ & $\begin{array}{l}\text { Rendimiento } \\
\text { (subió +, bajó -) }\end{array}$ & $\begin{array}{c}\text { CIT } \\
\text { WISC-IV }\end{array}$ \\
\hline 1 & 14 & 4 & 8 & 7.8 & -0.2 & 114 \\
\hline 2 & 14 & 18 & 7.7 & 6.9 & -0.8 & 102 \\
\hline 3 & 12 & 4 & 7.6 & 8.3 & +0.7 & 89 \\
\hline 4 & 10 & 9 & 8.1 & 5.8 & -2.3 & 66 \\
\hline 5 & 16 & 36 & 8.5 & 8.1 & -0.4 & 78 \\
\hline 6 & 14 & 6 & 7.3 & 8 & +0.7 & 81 \\
\hline 7 & 14 & 14 & 7.5 & 7.5 & 0 & 100 \\
\hline 8 & 12 & 44 & 9 & 9 & 0 & 105 \\
\hline 9 & 15 & 8 & 7.6 & 8 & +0.4 & 85 \\
\hline
\end{tabular}

Fuente: Elaboración propia. 
Tabla 3. Puntuaciones de los NyA en la WISC-IV (primera parte).

\begin{tabular}{ccccccccccc}
\hline ID & SE & VB & CM & IN & CV & DC & CD & MT & FI & RP \\
\hline 1 & 11 & 13 & 13 & 13 & 112 & 14 & 11 & 11 & 9 & 112 \\
\hline 2 & 13 & 12 & 10 & 12 & 108 & 11 & 11 & 9 & 9 & 102 \\
\hline 3 & 8 & 10 & 7 & 9 & 91 & 12 & 10 & 9 & 8 & 102 \\
\hline 4 & 6 & 6 & 1 & 6 & 67 & 4 & 5 & 6 & 5 & 69 \\
\hline 5 & 6 & 7 & 9 & 6 & 83 & 6 & 6 & 6 & 5 & 75 \\
\hline 6 & 7 & 8 & 7 & 9 & 85 & 7 & 9 & 9 & 7 & 90 \\
\hline 7 & 7 & 11 & 8 & 12 & 93 & 10 & 12 & 11 & 11 & 106 \\
\hline 8 & 12 & 13 & 10 & 13 & 108 & 13 & 11 & 9 & 7 & 106 \\
\hline 9 & 10 & 7 & 8 & 9 & 91 & 10 & 5 & 6 & 6 & 82 \\
\hline M & 8.9 & 9.7 & 8.1 & 9.9 & 93 & 9.7 & 8.9 & 8.4 & 7.4 & 94 \\
\hline
\end{tabular}

SE: semejanzas. VB: vocabulario. CM: comprensión. IN: información. CV: comprensión verbal. DC: diseño con cubos.

CD: concepto con dibujos. MT: matrices. FI: figuras incompletas. RP: razonamiento perceptual.

Fuente: Elaboración propia.

(con tres años y medio de diagnóstico) obtuvo calificaciones iguales, mientras que de los niños con menor tiempo (cuatro meses) uno bajó y el otro subió. En cuanto a la WISC IV, el coeficiente intelectual total del niño número 4 se ubica muy por debajo del promedio, mientras que los niños con los números 5 y 6 se ubican por debajo del promedio. Todos los demás niños se ubican en el promedio (Sueiro, 2005).

En la tabla 3 se presentan los puntajes obtenidos por todos los niños y adolescentes en la primera parte de la prueba de WISC IV (por razones de espacio, se dividió en dos).

Como se puede apreciar en la tabla 3, como grupo, los NyA obtuvieron puntajes promedio en los compuestos Comprensión verbal (que, entre otras, evalúa las habilidades de formación de conceptos verbales, expresión de relaciones entre conceptos, riqueza y precisión en la definición de vocablos [Lafourcade, 2018]) y Razonamiento perceptual (que valora el razonamiento perceptivo fluido, el procesamiento espacial y la integración visomotora [Lafourcade, 2018]). En el plano individual, los NyA identificados con los números cuatro y cinco obtienen los puntajes más bajos en los compuestos Comprensión verbal y Razonamiento perceptual. Los NyA con los números 1 y 8 obtienen los puntajes más altos, sin rebasar el promedio (en esta prueba, la $\mathrm{M}=$ 100 y la DE = 15).

En la tabla 4 se aprecia que, como grupo, los participantes obtuvieron un puntaje bajo en el compuesto Memoria de trabajo (la cual analiza la capacidad de retención y almacenamiento de información, de operar mentalmente con esta información, transformarla y generar nueva información [Lafourcade, 2018]). Este compuesto se asocia con el rendimiento académico (López, 2013) y un puntaje semejante al de otros compuestos en Velocidad de procesamiento (el cual evalúa la capacidad para focalizar la 
Tabla 4. Puntuaciones de los NyA en la WISC-IV (segunda parte).

\begin{tabular}{|c|c|c|c|c|c|c|c|c|}
\hline ID & $\mathrm{RD}$ & $\mathrm{NL}$ & $\mathrm{AR}$ & MT & $\mathrm{CL}$ & BS & $\mathrm{VP}$ & CIT \\
\hline 1 & 10 & 13 & 15 & 107 & 12 & 10 & 106 & 114 \\
\hline 2 & 14 & 10 & 12 & 77 & 5 & 7 & 78 & 102 \\
\hline 3 & 9 & 9 & 10 & 94 & 6 & 7 & 80 & 89 \\
\hline 4 & 7 & 6 & 4 & 80 & 7 & 4 & 75 & 66 \\
\hline 5 & 6 & 7 & 10 & 80 & 10 & 8 & 94 & 78 \\
\hline 6 & 3 & 7 & 8 & 71 & 9 & 9 & 94 & 81 \\
\hline 7 & 7 & 13 & 8 & 99 & 9 & 12 & 103 & 100 \\
\hline 8 & 7 & 11 & 10 & 94 & 10 & 10 & 100 & 105 \\
\hline 9 & 7 & 9 & 7 & 88 & 9 & 9 & 94 & 85 \\
\hline $\mathrm{M}$ & 7.8 & 9.4 & 9.3 & 88 & 8.6 & 8.4 & 92 & 91 \\
\hline
\end{tabular}

RD: retención de dígitos. NL: sucesión de número y letras. AR: aritmética. MT: memoria de trabajo. CL: claves.

BS: búsqueda de símbolos. VP: velocidad de procesamiento. CIT: coeficiente intelectual total. M: media

Fuente: Elaboración propia.

atención, explorar, ordenar y/o discriminar información visual con rapidez y eficacia [Lafourcade, 2018]). En el plano individual, y con excepción de los participantes identificados con los números 2, 4 y 6, cuyos puntajes del compuesto Memoria de trabajo fueron los más bajos, y los participantes con los números 2 y 4 , cuyos puntajes en el compuesto Velocidad de procesamiento también fueron los más bajos, el resto de NyA obtuvieron puntajes promedio. La niña identificada con el número 4 obtuvo el CIT más bajo.

Por otro lado, para conocer cómo es la integración social del NyA con DM en el contexto escolar, se consideró la opinión de los padres, docentes y compañeros. Siete de los padres de familia ( $\operatorname{casos} 1,3,5,6,7,8$ y 9) opinan que su hijo tiene una buena integración escolar, uno (caso 4) considera que la integración es regular y uno más (caso 2) que es mala. Referente a los docentes, tres consideran que la integración del alumno con sus compañeros es muy buena (casos 1, 7,9), dos consideran que es buena (casos 5 y 8), tres que es regular ( $\operatorname{casos} 3$, 4 y 6 ) y uno que es mala (caso 2). En relación con los compañeros de aula, de uno (caso 1) se opina que es muy buena, de siete (casos 3, 4, 5, 6, 7, 8 y 9) se considera que la integración de su par con DM es buena, y en uno más (caso 2), que es regular.

En este sentido, desde la óptica de los padres, docentes y compañeros, se podría señalar que la integración de los NyA en la escuela tiende a ser buena. Sin embargo, en los casos 2 y 4 no siempre es así, sobre todo en el caso 2 , quien presenta dificultades en la interacción con sus pares. Entonces, se puede decir que dos de los alumnos presentan NEE. Se trata de los casos 2 y 4.

El caso 2 corresponde a una alumna de 14 años que cursa tercero de secundaria, con diagnóstico de DM2. Enfrentó dificultades para ingresar a la institución educa- 
tiva, pues se trata de una escuela con mucha demanda, ya que tiene cierto prestigio de ser muy exigente. Pese a obtener una puntuación promedio en la WISC-IV, tiene cuatro de nueve materias reprobadas en su boleta de calificaciones (promedio de 6.9). Además se constató que presenta dificultades de integración escolar social, pues presenta problemas de incontinencia urinaria que, de acuerdo con su madre, junto con la DM fueron detonadas por una pancreatitis; el mal olor que despide ha provocado cierto distanciamiento con sus compañeros de aula. Por otro lado, la escuela negó tener alumnos con NEE, de lo que se podría inferir que, cuando estas se presentan, no las identifica ni atiende.

El caso 4 corresponde a una alumna de diez años que cursa quinto de primaria. Se observó que su ritmo de trabajo se encuentra desfasado con respecto al del resto del grupo, de manera que sus actividades quedan inconclusas, además tiene dificultades para cumplir con algunas actividades académicas (por ejemplo, para la realización de exámenes) debido a que falta con frecuencia a clases por su asistencia frecuente a consultas médicas (también asiste al servicio de nutrición y de psicología). Su promedio de calificaciones es de 5.8 (antes del diagnóstico tenía 8.1). Esto coincide con sus resultados en la WISC-IV, donde su puntuación de CIT está muy por debajo del promedio. Su integración escolar es considerada como regular. Asimismo, se observó que la escuela y algunos docentes no ofrecen apoyos para sus NEE.

Los resultados del cuestionario Estrategias para Fortalecer el Aprendizaje (que se pueden apreciar en la figura 1) muestran que los docentes de los NyA con diabetes se autovaloran muy alto en la subescala Estrategias de enseñanza (que involucra el apoyo a los alumnos para que definan metas y la enseñanza explícita, directa y sistemática de estrategias cognitivas). En las subescalas Estrategias de colaboración interinstitucional

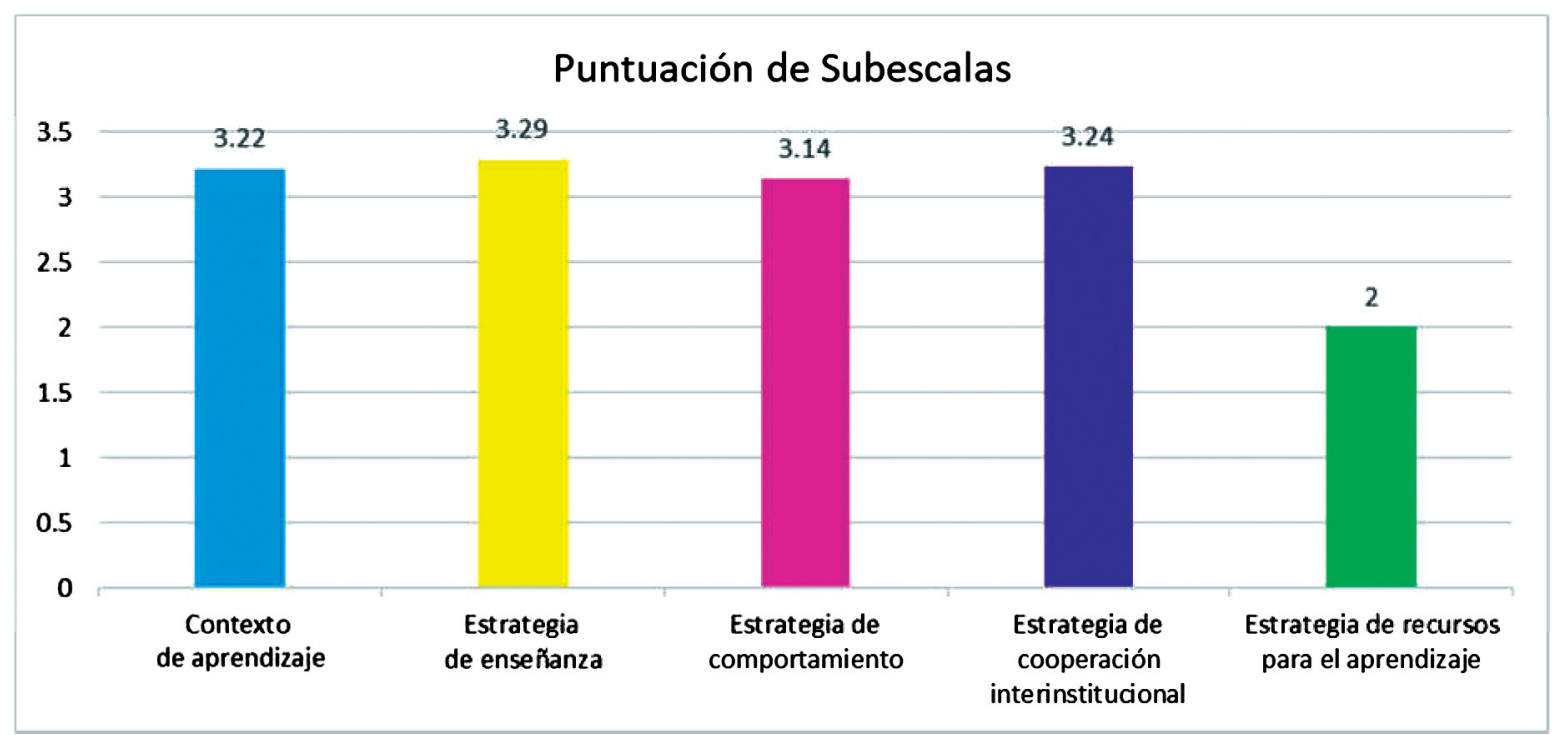

Figura 1. Resultados obtenidos por los docentes de los alumnos en el cuestionario de estrategias para favorecer el aprendizaje. Fuente: Elaboración propia. 


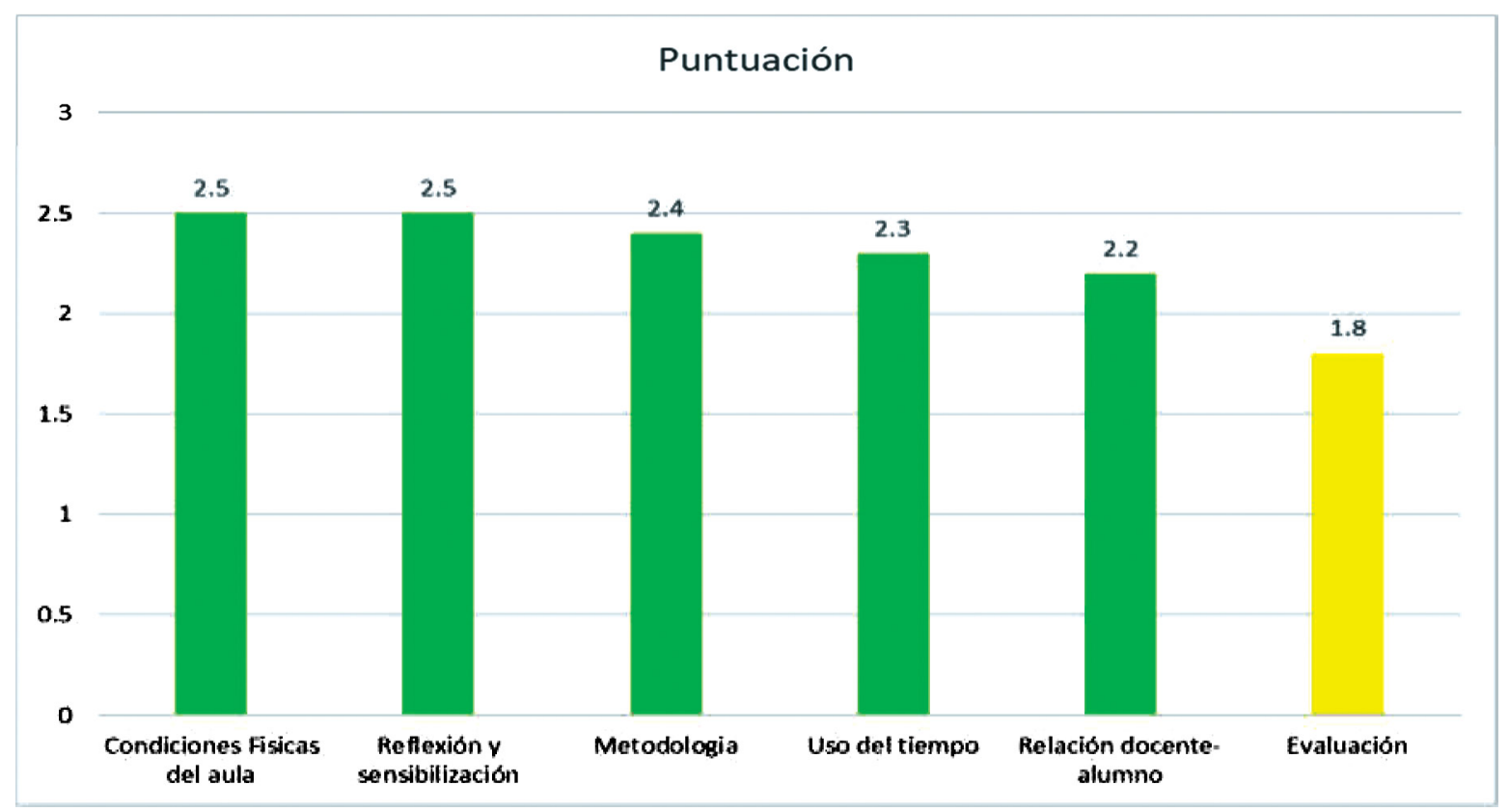

Figura 2. Resultados obtenidos por los docentes de los NyA en la Guía de Observación de Prácticas Inclusivas en el Aula- Formato de Observación (GEPIA-O).

Fuente: Elaboración propia.

(seguimiento del progreso de los alumnos, adaptaciones curriculares, uso del diseño universal de aprendizaje), Contexto de aprendizaje (implementación de la educación inclusiva, trabajo colaborativo y clima de aula) y Estrategias de comportamiento (uso de técnicas conductuales para el manejo de conductas disruptivas, enseñanza de habilidades sociales) se valoran un poco menos alto. Finalmente, en la subescala Recursos para el aprendizaje (uso de las tecnologías de la comunicación, apoyo de profesionales) se valoran de manera negativa. Los docentes de las participantes 2 y 4 obtienen puntajes muy cercanos al puntaje promedio de la muestra de docentes.

Finalmente, los resultados obtenidos en la GEPIA-O muestran un panorama un tanto distinto (figura 2). De acuerdo con la observación realizada por los investigadores, los docentes de los NyA con diabetes puntuaron en el promedio en dos subescalas, se trata de Condiciones físicas del aula (que valora cómo se utilizan los recursos dentro del aula, por ejemplo el material didáctico y el mobiliario) y Reflexión y sensibilización (empleo de estrategias, por parte del docente, para propiciar la participación de todo el alumnado), en tres puntuaron un poco por debajo del promedio, la subescala de Metodología (evalúa cómo presenta el docente los contenidos de aprendizaje y qué estrategias usa para desarrollar las competencias de sus alumnos), Uso del tiempo (cómo distribuye y administra su tiempo para la enseñanza) y Relación maestro-alumnos (si el profesor promueve relaciones de convivencia entre sus alumnos y de ellos con él) y dieron un puntaje negativo en la subescala de Evaluación (identifica si la evaluación es continua y formativa). La docente de la niña 4 obtuvo puntajes muy cercanos 
al promedio de sus compañeros. Por otro lado, debe aclararse que la dirección de la escuela no dio autorización para realizar la observación de la docente de la niña identificada con el número 2.

\section{Discusión}

De acuerdo con López y Valenzuela (2015), un estudiante presenta NEE cuando no alcanza los aprendizajes esperados para su edad y grado, por lo que necesita que se le proporcionen apoyos adicionales. Por su parte, Grau (2003) menciona que las dificultades de aprendizaje relacionadas con las enfermedades crónicas pueden asociarse a) al diagnóstico y tratamiento de una enfermedad, b) a los problemas emocionales y sociales asociados a la misma o c) a las metodologías promovidas por el currículo escolar.

A partir de los resultados obtenidos en el presente estudio se observa que, del grupo conformado por nueve casos, solo dos NyA probablemente presentan NEE (casos 2 y 4), aunque estas no parecen estar directamente relacionadas con el diagnóstico y el tratamiento de la DM. Estas dos participantes: a) obtuvieron las calificaciones escolares más bajas de la muestra; b) mostraron la mayor disminución de su calificación a partir del diagnóstico; c) obtuvieron puntajes bajos en el compuesto Memoria de trabajo de la prueba de WISC, compuesto fuertemente asociado al rendimiento escolar; d) en opinión de sus padres, maestros y compañeros, la niña de 14 años tiene una mala integración escolar, mientras que la niña de diez años tiene una integración mala, de acuerdo con su madre, y regular, de acuerdo con su maestra y compañero. Los siete participantes restantes no muestran indicadores de necesidades educativas especiales.

Vale la pena mencionar que las NEE de los alumnos no parecen estar asociadas con el tiempo que ha pasado desde el diagnóstico de DM. El niño que lleva más tiempo diagnosticado (tres años y medio) no presenta disminución en sus calificaciones, su CI total es promedio, él obtuvo un puntaje promedio en el compuesto Memoria de trabajo, su madre considera que tiene una buena integración escolar, mientras que un maestro y un compañero la consideraron regular. Por otro lado, en relación con los niños con menor tiempo de diagnóstico, en un caso bajó ligeramente su calificación (de 8 a 7.8) y en el otro la subió (de 7.6 a 8.3), su puntaje en Memoria de trabajo es promedio y sus padres consideran que tienen una buena integración escolar, mientras que en un caso su maestro y compañero la consideran buena y en el otro caso la consideran regular.

Las NEE tampoco parecen tener relación con el control de su enfermedad. Las participantes que probablemente presentan NEE alcanzan valores de la hemoglobina glucosilada normales (5.5 y 7.7, respectivamente). Con respecto al IMC, la niña de diez años que probablemente presenta NEE (caso 4) es una de las tres cuyo valor es promedio, mientras que la niña del caso 2 presenta obesidad. 
A pesar de que en el cuestionario Estrategias para Favorecer el Aprendizaje la mayoría de los docentes participantes en este estudio refirió realizar prácticas de inclusión, lo observado no concuerda con sus opiniones. De hecho, en la GEPIA se encontraron prácticas poco inclusivas en cuanto a la metodología empleada, el uso del tiempo, la promoción de relaciones cordiales entre alumnos y entre profesor y alumnos y en cuanto a los procesos de evaluación de los aprendizajes. Por lo anterior, se necesitan mayores esfuerzos para ser consistentes en dichas prácticas, pues es un reto importante brindar los apoyos necesarios al alumnado de grupos vulnerables (García, 2018).

En relación con toda la muestra, el promedio del CIT de la WISC-IV fue de 91, que se ubica en el promedio. Este resultado concuerda con los hallazgos de Fernández, Pérez y Arias (2010), quienes no encontraron diferencias significativas en los niveles de CIT de NyA con DM. Algunos autores, como Villa Martín (2011), refieren que los niños con enfermedades crónicas tienen peores resultados en matemáticas, sin embargo, los resultados de este estudio no concuerdan con sus afirmaciones (el valor del subtest de aritmética fue promedio, de 9.3). Como menciona Grau (2003), las enfermedades crónicas (como la DM) generan una situación de crisis a nivel personal y familiar, lo cual puede tener consecuencias en el contexto escolar, sin embargo, el mismo autor añade que dichas consecuencias dependerán de la reacción de la familia y del niño para adaptarse a vivir con la enfermedad.

En el estudio también se encontró que cuatro de los nueve NyA (casos 3, 5, 6 y 8) presentan niveles altos de hemoglobina glucosilada. Llama mucho la atención que esta situación no esté afectando a los $\mathrm{NyA}$ en su rendimiento académico, pues estos valores hablan de poca adherencia al tratamiento. Se tendría que investigar si la afectación mencionada se hace evidente al agregar la variable tiempo de permanencia de dichos valores altos.

Cabe señalar que existe cierta reticencia por parte de los padres y madres de familia y NyA para comunicar a la escuela sobre la enfermedad, a pesar de que con esta información podrían tomarse medidas preventivas o de cuidado; por ejemplo, los compañeros de aula podrían apoyar al NyA a controlar la enfermedad, o los docentes podrían hacer adecuaciones curriculares en la realización de algunas actividades y tareas.

Algunos de los posibles apoyos que precisan los NyA para cubrir sus NEE son a) que la escuela cuente con docentes bien informados, para que tomen en cuenta los apoyos que precisan los alumnos, entre ellos tolerancia frente a sus inasistencias, enviando tarea para que se haga en casa; b) la colaboración de personal de apoyo (maestro de apoyo, trabajadora social y psicólogo), y c) de adaptaciones de los métodos del currículo y de evaluación para satisfacer las NEE de todo el alumnado, entre ellos el que presenta DM. 
En conclusión, siete de los nueve NyA fueron identificados como alumnos sin NEE, pues han logrado calificaciones escolares cercanas o superiores a 8 , lo que indica un rendimiento académico promedio. De ellos, dos tienen puntaje por debajo del promedio y cinco puntajes promedio en el WISC IV, además sus padres, maestros y pares consideran que están bien integrados en sus escuelas. Por otro lado, se identificaron dos alumnos con NEE (casos identificados como el 2 y 4 ) y se encontró que sus NEE no parecen relacionarse directamente con la DM, además se encontró que dichas NEE no están siendo atendidas por sus escuelas y maestros. En ambos casos, la escuela y los docentes utilizan pocas estrategias para satisfacer sus necesidades. En el caso de la participante 2, por ejemplo, su dificultad de integración no estaba siendo atendida por ningún docente ni tampoco por la psicóloga de la escuela, además, como ya se mencionó, académicamente le estaba yendo mal y tampoco recibía apoyo pedagógico. Por su parte, el director de la escuela negaba tener alumnos con NEE y no autorizó la aplicación de la GEPIA. La mamá de la participante 4, por otro lado, tuvo citas en el hospital durante la semana de exámenes escolares. La escuela se negó a reprogramárselos, lo cual muestra su falta de apoyo a la alumna.

Finalmente, preocupa que los NyA presenten niveles altos de hemoglobina glucosilada y de IMC; al parecer sus familias no los están apoyando lo suficiente para que alcancen y mantengan una buena adherencia terapéutica. Urge hacerlo, pues de otra manera las consecuencias futuras para la salud (y para el desempeño académico) de estos NyA podrían ser muy dañinas. Entonces, urge que la escuela o el hospital promuevan en las familias de estos NyA su adherencia terapéutica.

\section{REFERENCIAS}

Acedo, C. (2008). Educación inclusiva: superando los límites. Perspectivas. Revista Trimestral de Educación Comparada, 38(1), 5-17.

Adame, V. (2015). Actitudes del profesorado ante el alumnado con necesidades educativas especiales [Trabajo de grado]. Badajoz, 2014/2015. Recuperado de: http://dehesa. unex.es/bitstream/10662/3263/1/TFGUEX_2015_ Adame_Sirgado.pdf.

Ainscow, M. (2001). Comprendiendo el desarrollo de escuelas inclusivas. Recuperado de: https://oas.org/cotep/ GetAttach.aspx?lang=en\&cId $=493 \&$ aid $=728$.

Asociación Médica Mundial (2013[1964]). Declaración de Helsinski. Recuperado de: http://www.conbioeticamexico.salud.gob.mx/descargas/pdf/Declaracion_ Helsinki_Brasil.pdf.

Bazán Calvillo, J. (2012). Problemas psicológicos del niño diabético. Recuperado de: http://cdn.psiquiatria.com/ bibliopsiquis/handle/10401/5390.
Bilbao-Cercós, A., Beniel-Navarro, D., Pérez-Marín, M., Montoya-Castilla, I., Alcón-Sáez, J. J., y PradoGascó, V. J. (2014). El auto concepto y la adaptación a la enfermedad en pacientes diabéticos pediátricos. Clínica y Salud, 25(1), 57-65. Recuperado de: http://linkinghub.elsevier.com/retrieve/pii/ S1130527414700271?via =sd.

Cascón, I. (2000). Análisis de las calificaciones escolares como criterio de rendimiento académico. Recuperado de: https:/ / campus.usal.es/ inico/investigacion/jornadas/jornada2/comun/c17.html.

Chamorro, M., Badajoz, I., y Luzuriaga, C. (2002). Aspectos psicosociales de las enfermedades crónicas en NyA. Revista Hospitalaria, 4, 170. Recuperado de: http://www.revistahospitalarias.org/ info_2002/04_170_04.htm.

Chamorro, M. M., Martínez, I. L., y Tomás, C. L. (2002). Perfil psicosocial de niños y adolescentes con diabe- 
tes mellitus. Bol Pediatr, 42, 114-119. Recuperado de: http:/ /www.sccalp.org/documents/0000/0879/ BolPediatr2002_42_114-119.pdf.

FID [Federación Internacional de Diabetes] (2015). Atlas de la Diabetes de la Federación Internacional de Diabetes, IDF (7 ${ }^{a}$ edición). Recuperado de: https://www. fundaciondiabetes.org/general/material/95/avancenuevo-atlas-de-la-diabetes-de-la-fid-7-edicionactualizacion-de-2015.

FID [Federación Internacional de Diabetes] (2018). IDF Diabetes Atlas. Eight edition 2017. Recuperado de: http://fmdiabetes.org/wp-content/ uploads/2018/03/IDF-2017.pdf.

Fernández, M. L., Pérez, J. P., y Arias, R. M. (2010). Evaluación neuropsicológica de la diabetes mellitus tipo 1 en la infancia. Recuperado de: http:/ / www.sciencedirect. com/science/article/pii/S1134323010650090.

García, I. (2015). Integración educativa o educación inclusiva, ¿qué conviene a México? Cátedra Cumex de Psicología. En A. García y O. Cruz (2015). Los retos de la psicologia en la sociedad contemporánea. Tuxtla Gutiérrez, Chiapas, México: Universidad de Ciencias y Artes de Chiapas.

García, I. (2018). La educación inclusiva en la Reforma Educativa de México. Revista Nacional e Internacional de Educación Inclusiva, 11(2), 49-62.

García, I., Escalante, I., Escandón, M. C., Fernández, L. G., Mustri, A. y Puga, I. (2000). La integración educativa en el aula regular: principios, finalidades y estrategias. México: Fondo Mixto México-España-Secretaría de Educación Pública.

García, I., Hernández, B. Y. y Silva, G. (2019). Necesidades educativas especiales del estudiantado infantil y juvenil que vive con VIH. IE Revista de Investigación Educativa de la REDIECH, 10(19), 121-138. Recuperado de: https://www.redalyc.org/jatsRepo/5216/521658239008/movil/index.html.

García, I., Romero, S., y Escalante, L. (2011, nov.). Diseño y validación de la guía de evaluación de las prácticas inclusivas en el aula GEPLA. Documento presentado en el XI Congreso Nacional de Investigación, Ciudad de México. Recuperado de: http://www.comie. org.mx/congreso/memoriaelectronica/v11/docs/ area_14/1390.pdf.
García, I., Romero, S., Escalante, L., y Flores, V. J. (2018). Algunas propiedades psicométricas de las Guías para Evaluar Prácticas Inclusivas en el Aula. Revista Española de Orientación y Psicopedagogía, 29(2), 8-28. Recuperado de: http:/ / revistas.uned.es/index. $\mathrm{php} / \mathrm{reop} /$ article/view/23150/18558.

García, M. I., Fernández, M. y Medina, B. (2010). Enfermedades raras. Necesidades sociales y educativas en la edad escolar. INFAD Revista de Psicología, 3(1), 545-552. Recuperado de: https://www.redalyc.org/ pdf/3498/349832326059.pdf.

Gómez-Rico, I., Pérez-Marín, M., y Montoya-Castilla, I. (2015). Diabetes mellitus tipo 1: breve revisión de los principales factores psicológicos asociados. Anales de Pediatría, 82(1), e143-e146. Recuperado de: http://www.sciencedirect.com/science/article/pii/ S1695403314001799.

Grau, C. (2003). Necesidades educativas especiales derivadas de problemas crónicos de salud. En J. L. Gallego y E. Fernández, Enciclopedia de educación infantil (vol. II, pp. 781-804). Málaga: Aljibe. Recuperado de: http://roderic.uv.es/handle/10550/40969.

Grau, C., y González Ortiz, M. (2001). Atención educativa a las necesidades especiales, derivadas de enfermedades crónicas y de larga duración. Recuperado de: http://dialnet. unirioja.es/servlet/articulo?codigo $=1203275$.

Hawrylak, M. F., y Grau, C. (2014). Necesidades educativas, asistenciales y sociales especiales de los niños con enfermedades minoritarias: propuestas para una atención interdisciplinar. Revista Nacional e Internacional de Educación Inclusiva, 7(3), 97-124.

Hernández, R., Fernández, C., y Baptista, P. (2010). Metodología de la investigación (5a. ed.). México: McGraw-Hill.

Instituto Nacional de Salud Pública (2012). Encuesta Nacional de Saludy Nutrición 2012. Resultados nacionales. Recuperado de: https://ensanut.insp.mx/informes/ ENSANUT2012ResultadosNacionales.pdf.

Lafourcade, M. (2018). WISC IV. Descripción e interpretación. Montevideo: Editorial Basiliza. Recuperado de: https://www.editorialbasilisa.com/wisc-iv-descripcion-e-interpretacion\#: :text $=\mathrm{El} \% 20$ test $\% 20$ WISC $\% 2$ DIV $\% 20$ para,en $\% 20$ un $\% 20$ Cociente $\% 20$ Intelectual $\% 20$ Total. 
López, M. (2013). Rendimiento académico: su relacion con la memoria de trabajo. Revista Actualidades Investigativas en Educación, 13(2), 1-19. Recuperado de: https:/ / ri.conicet.gov.ar/bitstream/handle/11336/1389/ rendimiento-academico-relacion-memoria-de-trabajo-lopez.pdf? sequence $=1$ \&is Allowed $=\mathrm{y}$.

López Melero, M. (2012). La escuela inclusiva: una oportunidad para humanizarnos. Revista Interuniversitaria de Formación del Profesorado, 26(2), 131-160.

López, M., y Valenzuela, G. E. (2015). Niños y adolescentes con necesidades educativas especiales. Revista Médica Clínica Condes, 26(1), 46-51. Recuperado de: https://ac.els-cdn.com/S0716864015000085/1-s2.0S0716864015000085-main.pdf?_tid $=8 f b f c 6 f 2-7 b c d-$ 4797-be2a-7f35052de090\&acdnat $=1541028320$ d8a4d5c2403b7b41a5fb72ea162e43ad.

Manchón, M. G., Carrasco, J. G., Fernández, J. R., Fernández, T. D., y de Frías, E. G. (2009). Necesidades del niño escolarizado con diabetes mellitus. Visión de padres y profesores. Anales de Pediatría, 70(1), 4552. Recuperado de: http://www.sciencedirect.com/ science/article/pii/S1695403308000118.

McCarthy, A. M., Lindgren, S., Mengeling, M. A., Tsalikian, E., y Engvall, J. C. (2002). Effects of diabetes on learning in children. Pediatrics, 109(1), e9-e9. Recuperado de: http://pediatrics.aappublications.org/ content/109/1/e9.short.

Mitchell, D. (2014). Estrategias para fortalecer el aprendizaje: cuestionario para el profesorado (2015). En What really works in special and inclusive education: Using evidence-based teaching strategies (trad. T. Fletcher, I. García-Cedillo y S. Romero-Contreras, S.).

Montero, L. A. A. (1991). El informe Warnock. Cuadernos de Pedagogía. Recuperado de: http://files.educdiferencial.webnode.cl/200000029-e3c50e4bff/ informe-warnock.pdf.

Northam, E. A., Anderson, P. J., Jacobs, R., Hughes, M., Warne, G. L., y Werther, G. A. (2001). Neuropsychological profiles of children with type 1 diabetes 6 years after disease onset. Diabetes Care, 24(9), 1541-1546. Recuperado de: http://care.diabetesjournals.org/ content/24/9/1541.short.

Norwich, B. (2010). Can we envisage the end of special educational needs? Has special educational needs out- lived its usefulness? The Psychology o Education Review, 34(2), 13-21.

OMS [Organización Mundial de la Salud] (2016). Informe mundial sobre la diabetes. Resumen de orientación. Recuperado de: http://apps.who.int/iris/bitstream/handle/10665/204877/WHO_NMH_NVI_16.3_spa.pd f;jsessionid $=249 \mathrm{~B} 6 \mathrm{~B} 8282 \mathrm{D} 574 \mathrm{E} 30 \mathrm{~B} 65377 \mathrm{~A} 2 \mathrm{CAC}$ 4369? sequence $=1$.

Presidencia de la República (1986). Reglamento de la Ley General de Salud en Materia de Investigación para la Salud. Recuperado de: http://www.salud.gob.mx/unidades/ cdi/nom/compi/rlgsmis.html.

Quintero, E. M., y Melo, H. E. (2012). Caracterización de procesos cognitivos de memoria, lenguaje y pensamiento, en estudiantes con bajo y alto rendimiento académico. Diversitas, 8(1), 123-138. Recuperado de: http://revistas.usta.edu.co/index.php/diversitas/ article/view/115.

Ramos-Estrada, D. Y., García-Cedillo, I., López-Valenzuela, M. I., y Murillo-Parra, M. (2020). Validación de un instrumento de estrategias para fortalecer el aprendizaje. Revista Electrónica Educare, 24(1), 1-15. Recuperado de: https://www.redalyc.org/jatsRepo/1941/194162217006/index.html.

Sánchez-Escobedo, P. (2006). Escala Wechsler de Inteligencia para Niños-IV. Manual técnico. Versión estandarizada. México: El Manual Moderno.

SEP [Secretaría de Educación Pública] (2016). Nuevo Modelo Educativo 2016. Recuperado de: http:/ /www. gob.mx/modeloeducativo2016.

SEP [Secretaría de Educación Pública] (2017). Modelo educativo para la educación obligatoria. Recuperado de: https://www.gob.mx/cms/uploads/attachment/file/198738/Modelo_Educativo_para_la_ Educacio_n_Obligatoria.pdf.

SEP [Secretaría de Educación Pública] (2020). Estrategia Nacional de Educación Inclusiva. Acuerdo educativo nacional. Implementación operativa. Recuperado de: https://www. animalpolitico.com/wp-content/uploads/2019/11/ ENEI.pdf.

Secretaría de Salud (2019). Sistema Nacional de Vigilancia Epidemiológica. Ssitema único de información. Recuperado de: https://www.gob.mx/cms/uploads/attachment/ file/427448/sem01.pdf. 
Sueiro, M. J. (2005). Info WISC IV. Perfil e informe. Madrid: TEA Ediciones. Recuperado de: http://www.web.teaediciones.com/ejemplos/Ejemplo_InfoWISC-IV.pdf.

UNESCO (1994). Declaración de Salamanca y Marco de Acción sobre necesidades educativas especiales. UNESCO. Recuperado de: http://www.unesco.org/education/pdf/ SALAMA_S.PDF.

USAID (2013). Tablas de IMC y tablas de IMC para la edad, de niños(as) y adolescentes de 5 a 18 años de edad y tablas de IMC para adultos(as) no embarazadas, no lactantes $=19$ años de edad. Recuperado de: https:/ /www.fantaproject.org/ sites/default/files/resources/FANTA-BMI-chartsEnero2013-ESPANOL_0.pdf.
Villa Martín, C. E. P. (2011). Influencia de las enfermedades crónicas en el rendimiento académico y en la integración socio-escolar. Clave XXI. Reflexiones y Experiencias en Educación. Recuperado de: http:/ / clave21. es/files/articulos/F08_EnfermedadesCronicas.pdf.

Wechsler, D., Flanagan, D. P., y Kaufman, A. S. (2007). Escala Wechsler de inteligencia para niños-IV. El Manual Moderno.

Zumbado, E., Sandi, L., Vega, L., y Chavem, D. (2005). Valores de referencia para hemoglobina glicosilada en niños sanos, entre junio y octubre del 2004 Hospital Nacional de Niños. Revista Médica del Hospital Nacional de Niños, 40(1), 16-26.

Cómo citar este artículo:

García Cedillo, I., y Lozano Cabral, K. (2021). Necesidades educativas especiales en niños y adolescentes con diagnóstico de diabetes. IE Revista de Investigación Educativa de la REDIECH, 12, e866. doi: https://doi.org/10.33010/ ie_rie_rediech.v12i0.866. 